\title{
Knowledge and Information Management Tools in Architectural Dimensions
}

\author{
${ }^{1}$ Aravindhan K \\ ${ }^{1}$ Department of Computer Science and Engineering, SNS College of Engineering, TamilNadu, India. \\ 1aravindhan03@gmail.com
}

\author{
Article Info \\ Journal of Biomedical and Sustainable Healthcare Applications (http://anapub.co.ke/journals/jbsha/jbsha.html) \\ Doi: https://doi.org/10.53759/0088/JBSHA202101008 \\ Received 28 August 2020; Revised form 25 September 2020; Accepted 30 October 2020. \\ Available online 05 January 2021. \\ (C2021 Published by AnaPub Publications.
}

\begin{abstract}
A knowledge systems design is a layer of learning modeling approach that focuses on applying generic Artificial Intelligence (AI) application approaches to a specific category of problem-solving activities. The consequences of 3 major approaches of the architectural dimension for the development of Knowledge Engineering (KE) tools are discussed in this study. The methodology is shown by a hierarchy of KE tools to enable systems management and knowledge development at the architectural design dimension, as well as architecture for controlling uncertainty through reasoning about actions. This article discusses architecture-dimension tools for KE. Knowledge architecture is a way of tailoring conventional AI problem-solving strategies to a specific set of activities Architecture describes a certain kind of issue solution (e.g., diagnostic or reconfiguration) at a theoretical dimension above the application, indicating which parts of a problem class are fundamental to the issue and which are integration artifacts. An information system's design is a partial model in which certain choices are taken ahead of time to accommodate specific job characteristics. Many medical diagnostic systems, for example, evaluate data from the bottom up to identify "triggered" illness hypotheses, and then create top-down tasks to gather evidence for and against the hypotheses. Although it may be executed in a number of ways, the "trigger and acquire proof" cycle is an essential aspect of any design for the domain of clinical imaging activities.
\end{abstract}

Keywords - Artificial Intelligence (AI), Knowledge Engineering (KE), Personal Construct Psychology (PCP)

\section{INTRODUCTION}

Cognitive analysis is a branch of Artificial Intelligence (AI) that develops rules to connect to information in order to simulate a human professional's cognitive approach. It examines the framework of a job or choice in order to determine how a result is achieved. The computer may then develop a repository of problem-solving approaches and the ancillary information required for each, which can then be offered up as issues for it to analyze. The resultant software might then help with diagnostics, troubleshooting, and problem resolution on its own and as a back-up to a human operator. The goal of expert systems was to convert the problem-solving skills of human specialists into a computer that might take in same facts and reach the same result. The transmission mechanism was the dominant strategy in early knowledge discovery endeavors. It went out of favor, nevertheless, when developers learned that individuals' decision-making expertise is not always obvious. Humans rely on alternative sources of information that aren't always logically related to the job at hand. While many choices can be directly traced to prior experiences on what works, they also rely on alternative pools of understanding that aren't always clearly linked to the job at hand. Equivalent reasoning and unstructured thinking are better terms for a few of which CEOs and entrepreneurs refer to as gut instinct or intuition leaps. These patterns of thinking don't lend themself to clear, step-by-step tree structure, and they may need bringing in sources of information that seem to charge more to transform in and evaluate than they are worth. A modeling technique has taken the place of the transfer procedure. Rather than aiming to replicate a decision step-by-step process, knowledge design focuses on developing a system that can provide the same conclusions as an expert without pursuing the same route or accessing the same data sources.

This solves some of the problems associated with tracing down the knowledge utilized in nonlinear thinking, since the individuals conducting it are often unaware of the data they are using. The model is valid provided as the results are equivalent. It's possible to improve a model once it regularly approaches the human expert. Bad conclusions may be tracked down and debugged, and procedures that provide comparable or better results can be rewarded. Decisions assist software already includes knowledge analysis and design. Knowledge engineers work in a variety of sectors to advance human-like functions, such as the capacity of robots to identify faces and analyze what people say for meaning. The understanding architects may not completely comprehend how conclusions are derived when the model's complexity develops. Knowledge engineering will eventually go from designing systems that solve issues as well as people to doing so numerically better. Artificial intelligence, when combined with additional talents such as natural language processing (NLP) and face recognition, might be the finest waiter, financial advisor, or tour operator the world has ever seen.

Since they encourage the abstract concept of truly representative and computer simulation components at the frame dimension, architecture-dimension instruments for understanding technicians can enhance the productivity of framework innovation and expertise acquirement because: They enable the cognitive architect and expert to work together to design 
systems using a common architectural construction expression instead of the fundamental implementations (such as by enforcing a constraint on the different forms and valuations of components in the knowledge base). The notion of an organizational dimension has been used in latest projects on cognitive organizations. Dong and Tang [1] discovered a number of "general activities," such as sequential diagnostic and regular construction, for which task-specific description language and regulatory systems were developed. Nurmayanti, Jumadi, Wilujeng and Kuswanto [2] have developed a number of technical expertise systems based on frameworks that combine cognitive learning instruments with problemsolving techniques

Adamski and Saeed [3] have described the HERACLES design's heuristic classification method. Berger [4] separated an intellectual investigator's understanding, which is used to construct its behaviour, from the representation of knowledge, which defines how the information is represented in a symbolic representation, in his AAAI President's Mailbox on the dimension of comprehension. The application of cognitive engineering techniques in construction is investigated in this paper. We provide three opposing viewpoints on what the structural layer comprises, all of which are shown in the perspective of MU. In order to handle uncertainty, MU is a structural design for thinking about the implications of actions. We show how a high-dimension evaluation amounts to hierarchical planning of the tools meant for knowledge discovery that allows MU application development and effective learning. Finally, we go through some of the advantages of this knowledge discovery method. The sections in this paper have been organized as follows: Section II evaluates the relevant literature assumptions. Section III presents the methodology for the research. Section IV critically evaluates the research. Section $\mathrm{V}$ focuses on the methods and discussions of knowledge discovery. Finally, Section VI concludes the paper.

\section{LITERATURE REVIEW}

Dias [5] argues that following the necessity for a structured technique to construct knowledge-based systems, Knowledge Engineering (KE) was launched in the 1970s out of intelligent systems. Information architecture was initially described by researchers as the technique of condensing a big body of knowledge into a system of exact standards and facts. Researchers expanded this concept to incorporate the importance of learning more about the features of expertise in general, as well as how this information may be used to extracting professional knowledge in domain-specific circumstances. Because of its unstructured character, the practical implementation of intelligent machines to KE had a number of drawbacks, according to academics. Several efforts to circumvent this bottleneck have been attempted in the past, particularly in the domain of information elicitation. The original goal of information elicitation in the realm of software development was to impart expert information to the information engineer.

Christian [6] argues that despite some encouraging outcomes from prior studies, the problem of adapting AI technologies to emerging KBSs proved futile. Researchers blamed these failures on the tiny size of the KBSs that were constructed, making it impossible to test the viability of diverse methodological techniques. The problem's sophistication is directly related to the difficulties posed in the mid-1960s during the "application emergency," when conventional application software designs could not really ramp up to establishing and implementing large and viable software package for solving real-world problems, leading to the establishment of Software Development as a restraint. In a similar vein, the KE restraint was founded with the principal aim of converting the processes that occur in KBS building projects from an art to a practice of engineering, allowing for better assessment and detailed understanding implicated in developing and maintenance KBSs, as well as the development of appropriate methods, special equipment, and language groups for KBS building projects.

Yearwood and Stranieri [7] argue that expert networks were first used to segregate domain information from generic reasoning in order to create sets of information base rules. Numerous investigations in the late 1980s found drawbacks to this technique and advocated for the implementation of a methodical methodology to KE. Researchers pointed out that information may be acquired in a variety of ways that should be taken into account while tackling knowledge-based task. He claims that the information dimension, that is deeper than the representational dimension, tackles questions like as why systems or agent conducts an action that is not based on logic, symbols frame, or rule. These descriptions utilized by Investigators at the data dimensions have since become the foundational ideas on that KE is based, and have sparked various more studies like as Scientists in this area. Researchers discriminated between distinct categories of knowledge in knowledge-based systems, and in a subsequent research, they classified the common patterns employed in addressing knowledge-dimension issues using a heuristic categorization. Their results were then used to solve knowledge engineering difficulties involving task learning. The emphasis shifted from task knowledge to domains knowledge in the mid-1980s. With the widespread usage of ontology systems for describing knowledge, explicit knowledge has taken center stage.

ABLES [8] argue that the expanding usage of ontology for idea exchange in a dispersed knowledge field like the World Wide Web (WWWW) resulted in the creation of many modeling languages, notably the Ontological Web Languages (OWL), which is currently utilized in the semantics web. In addition, these modeling domain languages incorporate patterns similar to those seen in knowledge and skills. An information role is the role that a certain knowledge area plays in addressing problems.

Gadgil [9] argue that unlike the homogeneous process of reasoning used throughout conventional intelligent machines, whereby a large knowledge-based pertains to all, this aids in constructing the issue by imposing constraint on how a particular knowledge field may be used for the subject of rationale, thus growing the viability of the problem-solving procedure. Decisions, norms, and cases data are some types of knowledge roles typically employed in evaluation methods. In his analysis of KE methodologies, Researchers advocated Personal Construct Psychology (PCP), which was automated and improved by Researchers. PCP proposes a model for expressing, learning, and processing information that takes into account the unstructured character of human cognition. 
Borgmann [10] claims that PEGASUS, computerized software created by researchers, is better at encoding aspects of human cognition based on expert language into formal ideas and organized knowledge. Researchers went on to say that it may be utilized in teaching by enabling other instructors to use an expert's terminology in the same manner that the expert does. The main disadvantage of this approach is that it combines the psychological concepts employed in PCP with its rationales and systems ideas when creating a structure for KE. In his research on psychologists' attitudes regarding information sources from clients, researchers found no link between verbal reports and mental behavior. Additionally, PCP's hierarchy is founded on uniformity and presupposes a very formalized and idiosynchratic presupposition. This is incorrect under the concept of fuzzy system, which believes that there is no one "proper response" for all cases, i.e., no one size fits all solution.

\section{METHODOLOGY}

The analysis was impacted by the discovery parameters used for the study, which were defined by the concerns that this research attempted to solve. Regarding the scope and purpose of this study, the research topics encompassed five major subdomains of KE, including the expertise role paradigm, knowledge inference methodologies, and ontology methods used in expert systems for the building of knowledge-based systems (KBSs). The following are the study questions: a) what are the existing knowledge elicitation approaches in KE? b) What are the present approaches to KE methodology? c) How important is ontology in KE? d) How does KE differ from other disciplines in terms of specific features? And e) Why is SSM the most appropriate (efficient strategy for address ing KE challenges in a geographically dispersed and shared field context? Both publication and conference papers were individually obtained from numerous online sources and chosen based on the conditions given above, using a multi-stage manual filtering procedure with objective confirmation at each step, in order to perform this literature search.

\section{CRITICAL ANALYSIS}

\section{Dimensions of the Architecture}

There are three ways to look at structures, and each one offers different responsibilities for architecture dimensions instruments. The functional perspective, for instance, potentially depicts a system as the implementation of generic AI principles tailored to a certain problem-solving approach. The blackboard design, for instance, is well-suited to situations with noisy information and statistical kinds of proof when represented functionally. A cognitive systems design focuses on using weak approaches to tackle a certain number of difficulties. An infrastructure is a complete architecture that comprises definitions of intelligence representations formalisms, inference systems, and control techniques. Commercially accessible AI programming environment include many of the architectural elements, such as frames and rule structures. Architectures, on the other hand, are products created by the trained professional for specific objectives, not random combinations of various components. A computer system is defined by a third perspective of an infrastructure.

A knowledge system design exposes representation components above the dimensions of their implementations, similar to how Lisp offers primitives for symbolic manipulations that the developer may use without worrying about how they are implemented in hardware. The architecture offers a vocabulary for describing a system's behavior in terms that are familiar to a trained professional or expert. For instance, most medical diagnostic systems enable triggering, which is the process of making certain hypothesis "productive" when certain occurrences, such as input data, happen. To the professional, activation may be defined as "calling to mind a diagnosis." Implementation dimension primitives may be used to create the effect of triggering (such as providing triggered illnesses higher certainty influencers or the agenda priority).

However, the medium of cognitive representation is words like triggering, not their application. Interpretation and information development are aided by task-dimension terminology like these. Triggering is something that expertise architects, professionals, and individuals can all grasp without having to worry about how it's done. It's simpler to create a virtualized machine that uses activation as a primitive. In conclusion, the functional perspective of architecture focuses on the actions of the applications that use it. Knowledge representation, inference algorithms, and other architectural components are highlighted in the structural view. These perspectives are combined in a virtual machine, which is an abstraction tools purposed to suit the effective functional needs of the class of problem-solving endeavours. The next subsection explains how the interconnections of different points of view result in a knowledge engineering too organization.

\section{MU Design Tools}

In this sub-section, we present MU, an architectural for computers that effectively manage uncertainties, with the goal of demonstrating how the three architectural viewpoints impact the construction of cognitive design tools. MUM (Managing Uncertainty in Medicine), a strategy for arranging a sequence of diagnostic queries, tests, and therapies for disorders expressing chest and abdominal discomfort, inspired MU. MUM's main goal is to determine what to do when data is inadequate for diagnosis and therapy. MUM considers tradeoffs such as the costs of proof, the maximal usefulness of possible given information what is currently known, the impacts of therapies and the scientific proof they produce, and so on, in the same way that a physician would. MU represents the system for structuring the MUM-oriented architecture, which reasons about ambiguous circumstances and decide how to behave. Based on the functional perspective, MU's task is to effectively control uncertainties by considering the required measures.

The tasks necessitate understanding of the impacts of activities on a variety of objectives, including presenting proof for and against assumptions, reducing costs, and curing the illness. Structurally, MU has a large inferential network of assumptions, substantiation and transitional inferences, and doings that actually prove and provide remedy; a cognitive 
ability of improving inferences; inferential processes for spreading the impacts of proof in learning and memories; and the help for methodologies that select among an action. As a visual system, MU effectively facilitates KE (Knowledge Engineering) in the diagnostic terminologies like hypothesis and potential-evidence. Disease, for example, is a phrase that is tailored for various domains and further instantiation as distinct disorders like angina. In the creation of information engineering tools, the interactions of different viewpoints of the MU structure are visible. Table 1 depicts a tool architecture that aids in the construction of networks within MU. The model is economically licenced Artificial Intelligence (AI) development tool, which incorporates core AI programming methods including pattern-matching rule interpreter and message-passing, as well as implementation primitives like rules and frames. Table 1's bottom layer provides an architectural representation of the MU application. This group of application contemporaries that can effectively be instantiated to provide a wider behavioural variety is not a model for an architectural since no function description has been supplied or suggested.

The functional perspective of architecture dictates how implementation-dimension components and approaches are tailored to a certain kind of problem-solving. Inferential relationships between data, intermediary conclusions, and hypotheses are all functional prerequisites of MU. It should keep track of how much people believe in each of these things, determine where to concentrate its concentration (i.e., which items to look for proof for), and what evidence to look for. The first dimension's frames and slots are specified as assumptions and inferential connections at the 2 nd layer in Table 1. Inferential relations act as conduits for information via the inference network. Combining functions explain how evidence to support assumptions is combined when transmitted from subordinate nodes, and rules are utilized to apply them. A subset of the slot values of hypothesis and data-gathering operations are utilized as control parameters to assist decide the focus of attention. The frame system's demons ("activated values") and signal sending capabilities are used to build the value transmission mechanism. To summarize, the architecture's structure is built from the application elements to effectively match the various functional requirements of particular problem-solving approach, culminating in a virtual environment, or task-specific shells.

Table 1. Knowledge engineering tools hierarchy for MU architectural support

\begin{tabular}{|c|c|c|}
\hline $\begin{array}{ll}\begin{array}{l}\text { Dimension of } \\
\text { Tool }\end{array} \\
\end{array}$ & Object in the users' perspective & Application support \\
\hline $\begin{array}{l}\text { Knowledge } \\
\text { acquisition } \\
\text { interfaces }\end{array}$ & $\begin{array}{l}\text { Domain-based terminologies } \\
\text { Effectiveness of treatment, treatment, costs of } \\
\text { testing, criticality of illnesses, confirmation of } \\
\text { testing results, triggering of disease symptoms, } \\
\text { medical testing, questions, intermediate diagnosis, } \\
\text { diseases }\end{array}$ & $\begin{array}{l}\text { Knowledge-centered utility } \\
\text { Language-based editors and form filling } \\
\text { interface, inferential consistencies, graphic } \\
\text { display of interfacing networks }\end{array}$ \\
\hline $\begin{array}{l}\text { Shell (digital } \\
\text { machine) }\end{array}$ & $\begin{array}{l}\text { Task dimension construct } \\
\text { Hypothesis, intermediate conclusion, data-collected } \\
\text { action, inferential relationships, integrating } \\
\text { functions, controlling parameters, control protocols, } \\
\text { preference ranking from an action }\end{array}$ & $\begin{array}{l}\text { Task-oriented reasoning approaches } \\
\text { Valuation propagation components, predicates } \\
\text { relating to the condition of the inherent } \\
\text { networks, protocol-based planning, decision- } \\
\text { based support systems }\end{array}$ \\
\hline $\begin{array}{l}\text { KEE (artificial } \\
\text { intelligence } \\
\text { toolbox) }\end{array}$ & $\begin{array}{l}\text { Application primitive slot, frame, protocol, pattern } \\
\text { match languages, lisp function and object, graphic } \\
\text { and windows object }\end{array}$ & $\begin{array}{l}\text { Artificial intelligence programing } \\
\text { methodologies } \\
\text { Network graphers, windows systems, message } \\
\text { passing, demon invocations, assumption } \\
\text { maintenance, inherent methodology, rule } \\
\text { interpreters, knowledge-based records }\end{array}$ \\
\hline
\end{tabular}

An infrastructure is created to handle a group of activities, such as clinical reasoning, rather than a single activity. Architectural-dimension attributes are created by the trained professional and expert for a specific application, just as implementation-dimension primitives are created by the architectural engineer. MUM, the expert for the chest pains, is structured in MU framework, as shown in Fig 1. Inference connections are initialised by direct references between proof and summary, e.g. the proof connection between standardized angina and EKG results. The speculation is initialized as illnesses such as the classical angina, transition results are initialized as a cluster, e.g. exercise-caused pains and inferential connections are effectively instantiated as particular reference between inferences and substantiations e.g. the prospective evidence connections between the classical angina and EKG results.

The inference engine may create a knowledge-acquisition interfaces to assist elicit information in domain terms by combining architecture-dimension structures such as hypothesis with domain-dimension concepts such as illnesses. The instructional designer in the shell provides information about architecture-dimension keywords, which domain components in applications inherit. On top of the hierarchical, knowledge acquisition utilities leverage meta-knowledge-set concerning the components in the knowledge base to aid the users to construct syntactically precise and semantically rational knowledge base. MU presently provides form-filling operators for all bases of knowledge items, interactive features for learning combination functions, and limited consistency-checking capabilities. Toolkits for obtaining control knowledge in an interactive way are currently being developed. 


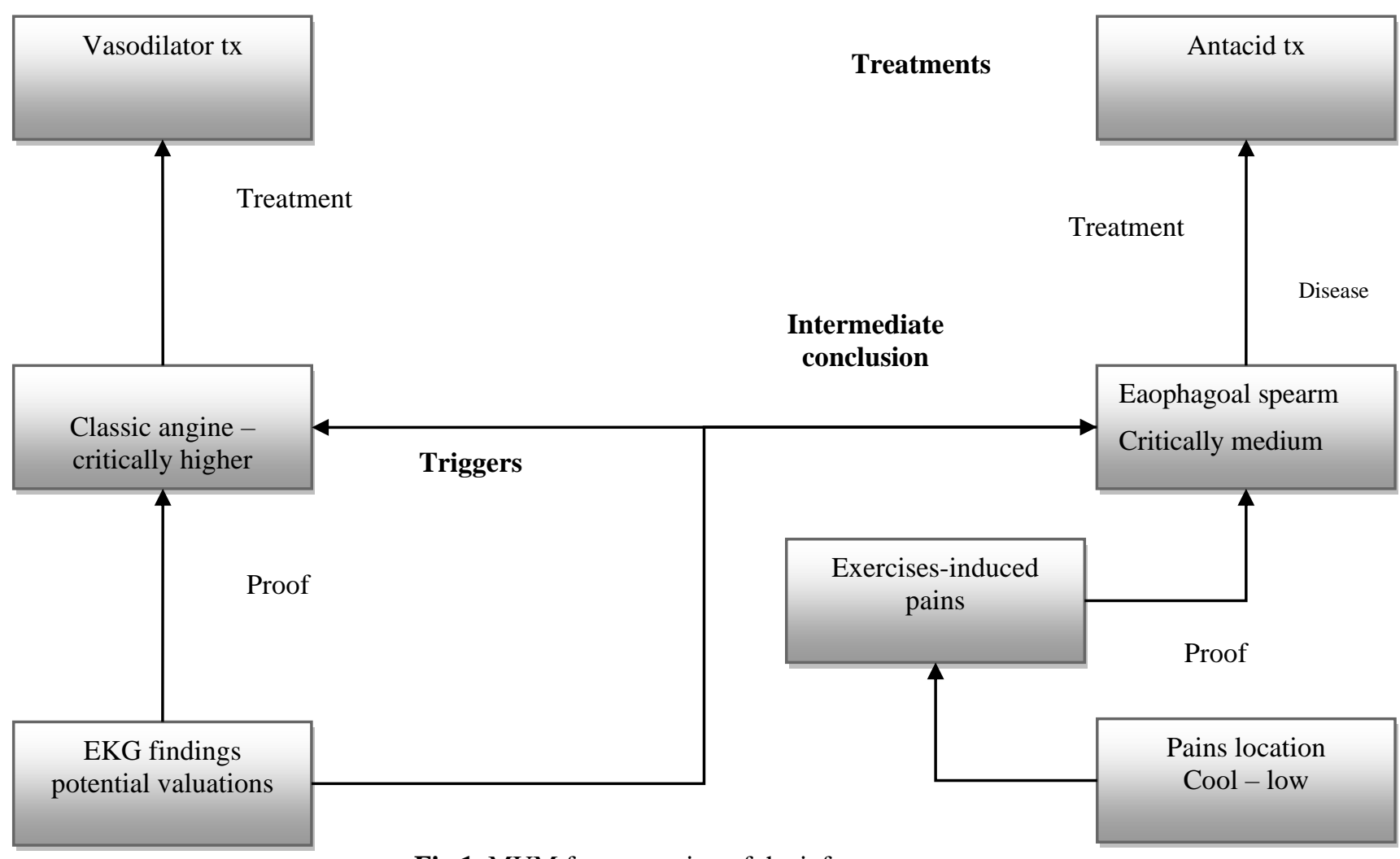

Fig 1. MUM fragmentation of the inference net

\section{METHODS AND DISCUSSION}

Expertise systems and databases use knowledge to solve complex issues. This information can be gathered from individuals or obtained autonomously using deductive, abductive, or inductive reasoning approaches. Instead of being encoded via sophisticated methods, this understanding is mostly expressed explicitly. This expressive presentation of understanding reduces the time it takes to design and maintain these technologies while also improving their readability. To calculate remedies for specified situations, knowledge-based computers initially relied on basic and general reasoning methods such as heredity and advanced or reverse resolutions. Nevertheless, for many real-world problems, this strategy proved to be impossible. Certainly, it differed with human analysts who solved their difficulties by utilizing understanding of the complexities of the problem-solving procedure. Researchers showed how expert architects incorporated explicit management knowledge by organizing manufacturing principles and assumptions of these regulations in such a way that the generalized inference mechanism displayed the dynamic characteristics they desired.

The reasoning that underpins Problem-Solving Methods (PSMs) is to make controlled expertise visible and treat it as a fundamental component of the more complete knowledge-set that has been stored by the knowledge-based systems. PSMs improve on the above-mentioned generalized inferences engine by allowing for more absolute authority of the chain of reasoning. Because this control information is defined irrespective of the application field, it is possible to reuse this operational understanding across domain and application. Furthermore, unlike conventional prediction machines that depends on a particular presentation of the information, PSMs abstraction from certain representational formalisms. Eventually, PSMs break down a knowledge-based program's thinking task into a series of subtopics and inference operations linked by learning responsibilities. As a result, PSMs are a subset of software components that describe the logical segment of the knowledge-based firms. Various problem-solving methodological frameworks are already accessible, and a variety of problem-solving methods description language, ranging from social transcriptions (e.g. CML) to explicit programming language, have been developed (e.g. KARL).

\section{Ontologies}

Ontologies Theories have become a prominent study subject, and numerous AI research societies, integrating interpretation of natural language, $\mathrm{KE}$ and information retrieval, have examined them. The concept of ontology has lately gained traction in sectors such as meaningful data and logic applications, as well as organisational learning. In these domains, ontologies fill a critical need: they create a universal and shared ideology of the subjects, which can effectively be conveyed across multiple machines and people. Various definitions of ontologies have been projected over the past few decades; however, we posit that the definitions in [11] effectively capture the fundamentals of ontologies. A clear and formal specification of shared comprehension is ontology. The conceptual framework represents abstract representations of the aspect of the world that is created by recognizing the necessary ideas. The term "explicit" refers to the types of concepts utilized and the limits that apply to their application. The term 'formal' alludes to the ideology that the ontologies have to be machines readable, with exclusion to natural languages; "shared" signifies the ideology, which the ecosystem records acceptable understanding, that is, knowledge that is not personal to a single person but approved by a group. 
Ontologies are used in the knowledge discovery approach to make the building of the domains framework easy. Ontologies are structures of terminologies and relationships that can be used to describe a domain. Diverse types of ontologies can be recognized depending on the dimension of complexity, and they can play various objectives in the structuring of knowledge-centred systems. We effectively identify the ontological kinds, among many others, below:

- Domain ontologies collect the knowledge that is applicable to a specific forms of domains (such as digital domains, mechanic domains, medical domains, and electronic domains)

- Common-sense ontology strives to effectively capture generalized comprehension of the globe and provide a foundational conceptions and ideology for the concept such as events, states, places and time. Resultantly, they are applicable to a variety of domains.

- Symbolic ontologies do not bind themself to a certain domain. Without specifying what should be expressed, such ontologies supply representation elements.

- The Frame Ontology is a well-known representation ontology that includes notions like restrictions of the slots, slots and the frames, permitting data architects to signify knowledge in the object-based or frame-based way. Part of the ontology study involves imagining and building a technology that allows for large-scale globalized re-application of ontology.

Ontology has to be compact elements with firm internalized coherence and little interactions between them in an attempt to allow as much reuse as feasible. This and other requirements are articulated in ontology design concepts, and handled by the ontolingua servers or SKC (Scalable Knowledge Composition) projects. PSMs and ontologies have been included into a number of modelling systems, including CommonKADS, MIKE, and PROTÉGÉ.

\section{Techniques and Methods}

Fundamental Methods and Techniques Given the vast quantity of data accessible at various information resources and the enormous number of them accessible via modern technology, such as the World Wide Web, retrieving, discovering, or analyzing data remains a tough endeavor. One of the key causes of this difficulty is the significant gap among the customer's (or one data programs) conceptual frameworks of data and the real preservation and supply of data by another data system. The challenge remains, for the most part, how to fill the loophole, and how to fulfil it in a way that potentially optimizes the technical works for big data and massive data sources. Whenever it comes to the establishment of the typical conceptualization of the prevailing data, free data and semi-structured datasets such as the XML dataset all face the same challenges. Domain ontologies, as stated before, are high-dimension technical abstractions that provide common conceptualizations for specific areas of interest. They identify key concepts, their connections, and the axioms that govern those relationships, ensuring that the conceptualization has a well-defined semantics. Domain ontologies provide the accurate definition of conceptualization typical to different sources of data in terms of information integration, sharing, and extraction.

As a result, ontologies offer partial remedies to the issues of extracting, exchanging and integrating datasets and communicating the same to clients or the centralized database entities in a clear and understandable manner. Since traditional ontology construction difficulties have been extensively explored and displayed in the past, we concentrate on how to reuse and change existing conceptual frameworks and problem-solving strategies, as well as how to effectively structure and maintain an ontological semi-automated manner based on the application of learning methodologies.

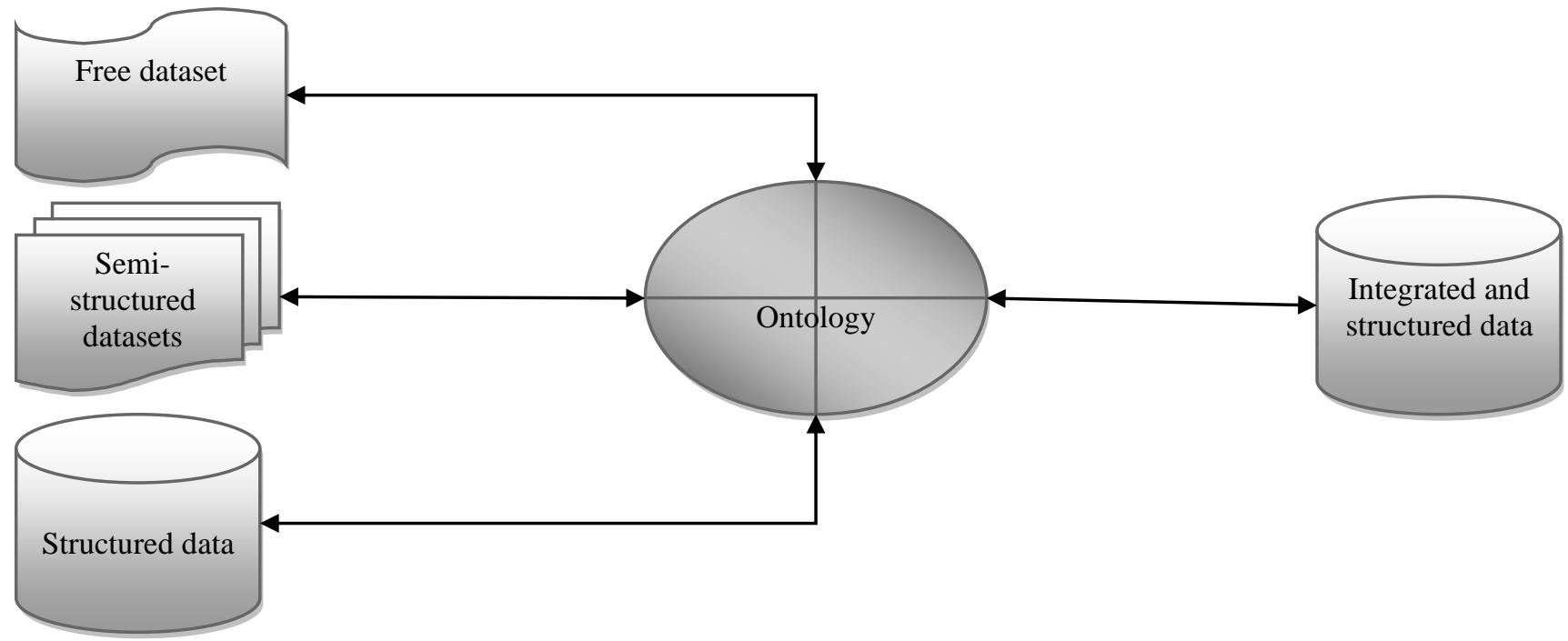

Fig 2. Data extraction and integration from the respective data exchanges etween several sources of data

\section{Information Synthesis and Retrieval}

Various information streams are now commonly integrated utilizing 2 dimensions of computer, such as database applications, text files, HTML files, and XML archives (TSIMMIS approach). The first dimension separates each source 
and generates accessibility methodology, which are agnostic of the resource architecture. The package permits users' queries and guidelines to be converted into the source-oriented queries. Perceptibly, not the resources support a similar form of research [13 -18]; for example, wrappers enclosing a storage device may only support a tiny subset of all SQL operations.

In recent decades, many methods for different storage types have been created, such like (rather) standardized information storage, HTML, or XML, and automatic wrapper generation has been investigated. Evidently, some platforms, such as Ontobroker, currently handle a more diverse set of information formats. Because human language documents convey a lot of important data, wrapper design starts to include data extraction and human language information retrieval technologies. Wrapper technology companies are now concentrating their efforts on conceptual parsing technologies, whereas human language syntax is mainly disregarded. All of these strategies use expertise frameworks, which integrate information from a variety of domains. These models define the entities that may be accessed from outside the wrappers, even if they are often quite simple and only exist in the heads of the designers. Many more algorithms rely on an expressive ontology, and some modern techniques explicitly explain data migration in elements of (ontological) declaratory rules. Wrappers provide standard file formats, while the second dimension comprises one or more intermediaries that bring together disparate data providers.

For instance, one provider might maintain managerial information from employees, while the other might keep track of their competence. The bundled materials are then combined by a facilitator to offer a query capability for personnel with specific knowledge who could be ready for a task at a specific moment. In order to complete this task, the facilitator must extract 'fused' entities, which have data from both the first and second sources. This fusion effort, on the other hand, necessitates an alignment of several knowledge-based systems, or to place it alternatively as an ontological convergence. Compatibilities of schemata have become a prevailing concern in the computed realm and language signals, as in ontological design in general, may help a lot in deciding which contenders for conceptual conformity are suitable. Nevertheless, above idea matching, ontological arrangement requires assistance. Ontologies, for example, often grow separately of one another, and if the origin abstractions change, an alignment may no longer operate appropriately. Sulis [12] suggest utilizing ontology algebra to build minimum ontology articulate in order to reduce this impact. While this looks to be a viable method, further study is needed to address a number of outstanding challenges, such as the interaction of theories with an axiom and semi-automatic synthesis and adoption of the concept for a novel application.

\section{Interchange Ontology}

Swapping expertise is a circumstance that is quite similar to the difficulty of obtaining and combining data from diverse sources (see Fig 2). Previously separated historical information management, expert systems, or when a data system involves information accessible to naïve individual in a comprehensible manner, e.g. the table and computer-produced message may all result in such circumstances. FIPA presented an ontology-based framework for multi-agent networks. Regardless of the fact that only a few multi-agent platforms use a comprehensive information model, ontologies are generally acknowledged as being critical for conceptually versatile and appropriate interaction between individuals in the coming years. Clearly, the main issue is once again the incorporation and convergence of much ontology, since in a heterogeneous network; one can only anticipate encountering another agency with a recognized, standardized ontology on a rare occasion. The present talks regarding expanding the W3C RDF approach represent this universal need for data and ontologies to be exchanged freely in an open setting, such as the World Wide Web (WWW).

\section{Reuse/Adaptation of Components}

For a long time, KE has focused on the recycling and adaption of real concern methodologies and ontologies. Structures like CommonKADS and PROTÉGÉ, in particular, have spent a lot of time and effort establishing ideas and techniques for components recycling. With the ease with which current elements may be accessed over the Internet, this field is gaining in popularity. The UPML framework integrates the majority of previous ideas and establishes a baseline for defining and transferring knowledge-based aspects of the system. It breaks down the full definition of a knowledge-oriented structure into 6 different segments (see Fig 3): a task, which categorizes the problem that the knowledge-based systems should resolve, a problem-solving technique that specifies the probabilistic reasoning, and subject models that specifies the domain knowledge. Each of these aspects is specified separately so that task specifications may be reused across domains, problem-solving techniques can be reused across tasks and domains, and domain knowledge can be reused across projects and problem-solving techniques.

The tools hierarchies illustrated here represent a compromise between power and flexibility. Frameworks at the implementation stage are broad (for example, development models may be modified to mitigate many issues), but they are poor in terms of knowledge representation. Even if the illness assumption' application may be nothing more than a frame, referring to an item as a disease assumption implies much more understanding about it than referring to it as a frame. This additional information restricts the illness frame's structural properties (e.g., the quantities and categories of spaces, or the sorts of signals it can receive, etc.), as well as its interactions with other frameworks. Architecture-dimension items such as illness frames are at the "strength" end of the influence spectra since these limitations promote knowledge development. Implementation-dimension objects are more generic but less strong in terms of knowledge engineering due to their absence of limitations. As a result, the effectiveness of an architectural dimension strategy while developing an intelligent system for a job is totally dependent on how much an individual comprehends about a specific task. The cognitive projected architecture encapsulates data concerning the category of problem-solving assignments - it is the virtualization initiative for that category - and so makes architecture formation and knowledge acquisition more easier for decision-makers in that 
category. We can alleviate the knowledge discovery bottleneck for limited classes of activities by developing designs and constructing incorporated "tools" at the architectural dimension, according to the influence of reasonable compromise.

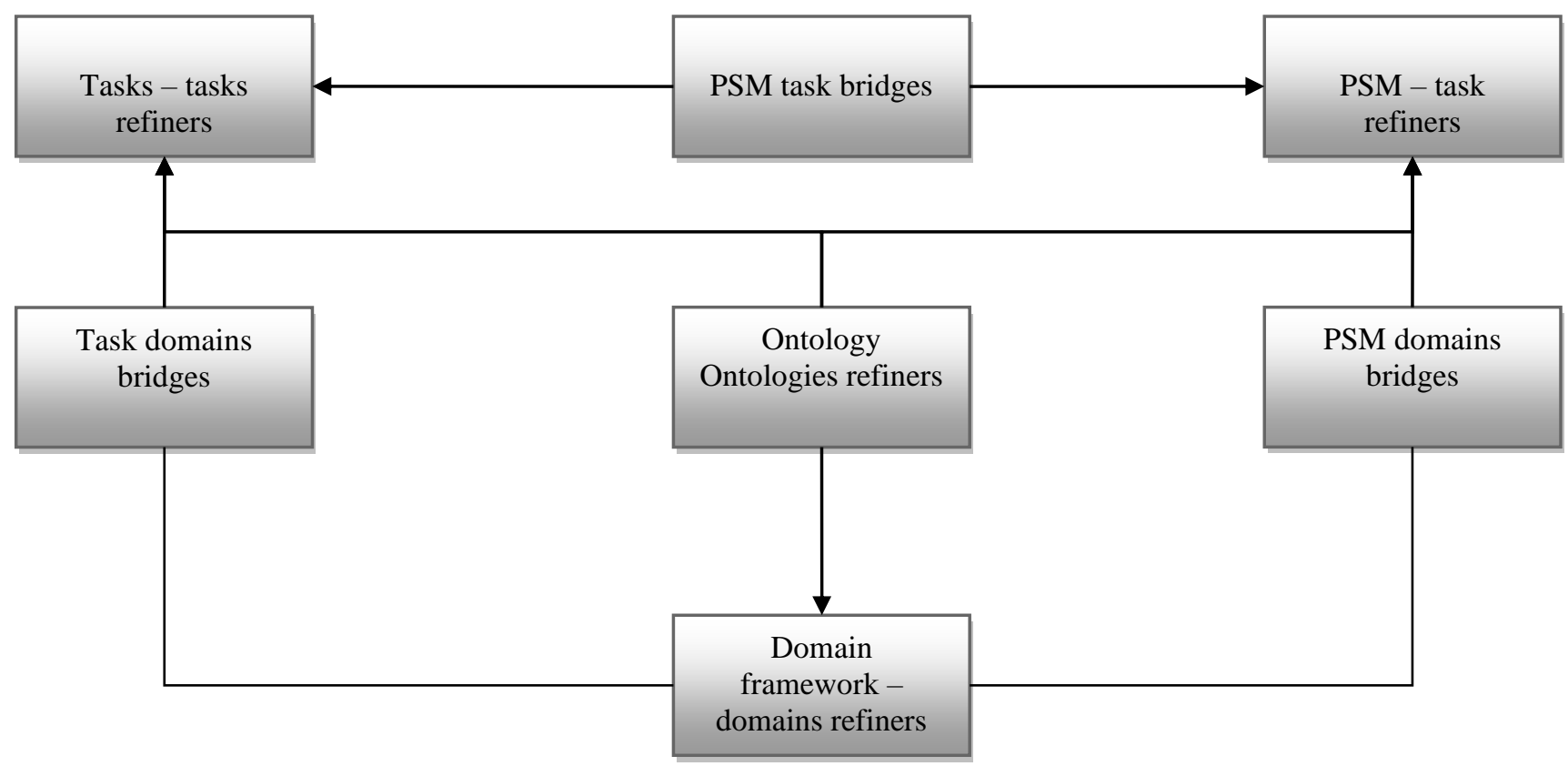

Fig 3. UPML structure for the knowledge-centred system

\section{CONCLUSION}

The following are some of the benefits of using architecture-dimension KE tools:

- Vertical embedding of application design might be beneficial at the architectural level. For instance, in the application dimensional, a general frame editors and networking grapher (like the KREME platform) may be configured as knowledge discovery functionality for editing system-level elements integrating such assumptions and implementations as illnesses. Because architecture-dimension items are generalizations of integration entities (i.e., frameworks), they share similar architecture.

- Declarative programming describing architecture-dimension structures - the virtual computer's basic components - promotes a unified design shared by a group of developers. Once the triggering relationship has been developed, for instance, there is no need to be concerned about many participants of a software system attempting to accomplish the same capability using various technologies.

- Because meta-knowledge may be connected to items to evaluate for coherence, give assistance, produce justifications, etc., prescriptive architecture-dimension components make explicit knowledge easier. For illustration, a form-filling system designed for obtaining an example of an illness may present a selection of recorded alternatives based on a descriptive characterization of the attributes of illnesses, such as the types of relationships they have with datasets.

The responsibilities of the trained professional and expertise are defined by constructing a virtual network at the architectural dimensions and then an information extraction layer on upper edge of the virtual environment. The expert implements architecture-dimension structures for the application area, while the instructional designer creates infrastructure by specialized general-purpose application design to optimize the constructions appropriate for the conflict resolution job. Cognitive learning tools enable the expert construct, revise, and troubleshoot the knowledge and understanding, while virtual mechanical devices aid the information designer in creating an architectural for a particular applications.

\section{References}

[1]. T. Dong and L. Tang, "Sequential diagnostic trial designs", Wiley Interdisciplinary Reviews: Computational Statistics, vol. 3, no. 1, pp. 79-83, 2010. Doi: 10.1002/wics.136.

[2]. D. Nurmayanti, J. Jumadi, I. Wilujeng and H. Kuswanto, "Developing of Learning Instruments based on Software Tracker in Measuring Cognitive Learning Outcomes", Journal of Physics: Conference Series, vol. 1233, p. 012047, 2019. Doi: 10.1088/1742-6596/1233/1/012047.

[3]. M. Adamski and K. Saeed, "Heuristic Techniques For Handwritten Signature Classification", International Journal of Computing, pp. 87-92, 2014. Doi: 10.47839/ijc.5.2.401.

[4]. S. Berger, "Meaning and understanding in intellectual history", Global Intellectual History, vol. 5, no. 3, pp. 329-354, 2020. Doi: 10.1080/23801883.2020.1729463.

[5]. W. Dias, "Philosophical grounding and computational formalization for practice based engineering knowledge", Knowledge-Based Systems, vol. 20, no. 4, pp. 382-387, 2007. Doi: 10.1016/j.knosys.2006.06.002.

[6]. G. Christian, "Predictive Coding: Adopting and Adapting Artificial Intelligence (AI) In Civil Litigation", SSRN Electronic Journal, 2020. Doi: $10.2139 /$ ssrn.3530039.

[7]. J. Yearwood and A. Stranieri, "Deliberative discourse and reasoning from generic argument structures", AI \& SOCIETY, vol. 23, no. 3, pp. 353-377, 2006. Doi: 10.1007/s00146-006-0069-y. 
[8]. T. Ables, "On The Very Idea Of An Ontology Of Communion: Being, Relation And Freedom In Zizioulas And Levinas", The Heythrop Journal, vol. 52, no. 4, pp. 672-683, 2010. Doi: 10.1111/j.1468-2265.2010.00630.x.

[9]. S. Gadgil, "Homogeneous Length Functions on Groups: Intertwined Computer and Human Proofs", Journal of Automated Reasoning, vol. 64, no. 4, pp. 677-688, 2019. Doi: 10.1007/s10817-019-09523-1.

[10]. N. Borgmann, "Pegasus \& Dragon - Die größte Pferdestatue der Welt aus Bronze", Stahlbau, vol. 85, no. 1, pp. 65-70, 2016. Doi: 10.1002/stab.201620353.

[11]. I. Jureta, J. Mylopoulos and S. Faulkner, "A core ontology for requirements", Applied Ontology, vol. 4, no. 3-4, pp. 169-244, 2009. Doi: 10.3233/ao-2009-0069.

[12]. W. Sulis, "An Information Ontology for the Process Algebra Model of Non-Relativistic Quantum Mechanics", Entropy, vol. 22, no. 2, p. 136, 2020. Doi: $10.3390 / \mathrm{e} 22020136$.

[13]. A. Haldorai and A. Ramu, "Security and channel noise management in cognitive radio networks," Computers \& Electrical Engineering, vol. 87, p. 106784, Oct. 2020. doi:10.1016/j.compeleceng.2020.106784

[14]. A. Haldorai and A. Ramu, "Canonical Correlation Analysis Based Hyper Basis Feedforward Neural Network Classification for Urban Sustainability," Neural Processing Letters, Aug. 2020. doi:10.1007/s11063-020-10327-3

[15]. D. Devikanniga, A. Ramu, and A. Haldorai, "Efficient Diagnosis of Liver Disease using Support Vector Machine Optimized with Crows Search Algorithm," EAI Endorsed Transactions on Energy Web, p. 164177, Jul. 2018. doi:10.4108/eai.13-7-2018.164177

[16]. H. Anandakumar and K. Umamaheswari, "Supervised machine learning techniques in cognitive radio networks during cooperative spectrum handovers," Cluster Computing, vol. 20, no. 2, pp. 1505-1515, Mar. 2017.

[17]. H. Anandakumar and K. Umamaheswari, "A bio-inspired swarm intelligence technique for social aware cognitive radio handovers," Computers \& Electrical Engineering, vol. 71, pp. 925-937, Oct. 2018. doi:10.1016/j.compeleceng.2017.09.016

[18]. R. Arulmurugan and H. Anandakumar, "Early Detection of Lung Cancer Using Wavelet Feature Descriptor and Feed Forward Back Propagation Neural Networks Classifier," Lecture Notes in Computational Vision and Biomechanics, pp. 103-110, 2018. doi:10.1007/978-3319-71767-8_9 